\title{
16 IS GENDER OF HEALTHCARE PROVIDER IMPORTANT FOR SCREENING FOR INTIMATE PARTNER VIOLENCE AGAINST WOMIN? EXPERIENCES FROM A NIGERIAN SAMPLE
}

doi:10.1136/injuryprev-2012-040590q.16

Ime Akpan John. Society Of Nigerian Doctors for The Welfare Of Mankind (SNDWM), Kano, Nigeria

Background Intimate partner violence (IPV) against women is gendered based violence that has varying degrees of health consequences on women. Advocacy for screening for IPV within healthcare and elsewhere has been championed for several years. Despite these calls, identification of IPV within the healthcare has met with challenges that relates to occupational and demographic characteristics of the Healthcare provider (HCP) especially gender.

Objectives To study the association between genders of HCP and screening for IPV at Aminu Kano Teaching Hospital Kano, Nigeria.

Investigate the association of gender with the readiness to screen for example, perceived self-efficacy, access to social network to refer victims of IPV, professional roles, attitudes towards women and safety of provider /Victims.

Method A cross-sectional questionnaire study using the Domestic violence healthcare provider survey scale. T-test was utilised to assess the association between inquiry for violence and readiness to screen variables. Associations between categorical variables were assessed using $\chi^{2}$ test.

Results Of the 693 clinical staff at the hospital, 430 had regular contact with patients. $90 \%$ of nurses were female while $70 \%$ of doctors were males. Inquiry for IPV among both genders was $25 \%$ and the males HCP inquired more than their female peers. Male HCP were more likely to blame the victims of IPV than the females. However, the females scored higher in the professional role resistance. 
Significance In our study, gender of HCP was important in the diagnosis and management of IPV. Screening was low but increased inquiry was associated with the male gender. 